\title{
Contribución al conocimiento de los Acanthosomatidae (Hemiptera: Heteroptera) de la Región de Magallanes, con descripción de una nueva especie
}

\author{
Contribution to the knowledge of the Acanthosomatidae \\ (Hemiptera: Heteroptera) of the Magallanes Region, \\ with description of a new species
}

Mariom A. Carvajal ${ }^{1,2}$, Eduardo I. Faúndez ${ }^{1,2}$

\& David A. Rider ${ }^{1}$

\section{Resumen}

Se describe Planois smaug sp. nov. de Isla Navarino, Región de Magallanes, y se entrega una lista actualizada de los Acantosomátidos de la Región de Magallanes, indicando su distribución por provincias. Se añade una clave de identificación para las especies de la familia presentes en Magallanes.

\section{Palabras clave:}

Heteroptera, Acanthosomatidae, nueva especie, lista, clave de identificación.

\begin{abstract}
Planois smaug sp. nov. is described from Navarino Island, Magallanes Region, Chile. An updated checklist of the Acanthosomatidae of Magallanes is provided, including province distributions. A key to de identification of Magallanes acanthosomatids is provided.
\end{abstract}

Key words:

Heteroptera, Acanthosomatidae, new species, checklist, identification key.

\section{INTRODUCCIÓN}

Los Acantosomátidos son una familia de Pentatomideos fitófagos que ha pasado recientemente por constantes cambios taxonómicos; actualmente contiene aproximadamente 230 especies en 56 géneros (Tsai \& Rédei, 2015). En Sudamérica Acanthosomatidae se encuentra representada por 33 especies en 19 géneros. A pesar de que la biología de esta familia en Sudamérica no ha sido estudiada en profundidad, se sabe que la mayoría de sus representantes se caracterizan por su capacidad de cambiar de color; estos cambios son estacionales, y estarían controlados por el fotoperíodo y la temperatura (Faúndez \& Osorio, 2010).

Durante la última década el conocimiento de los Acantosomátidos de la Patagonia y especialmente aquellos en Magallanes, ha incrementado considerablemente. Faúndez (2007a) y Faúndez et al. (2009) describen los hábitos sexuales y comportamiento gregario de Ditomotarsus hyadesi Signoret, 1881 (en ese entonces Ditomotarsus punctiventris Spinola, 1852), seguido por Carvajal y Faúndez (en prensa) que describen el ciclo de vida completo de la especie, y entregan nuevos datos biológicos para ésta. Asimismo la biología y cambios de color en Sinopla perpunctatus Signoret, 1864 han sido estudiados por Faúndez (2007b) y Faúndez y Osorio (2010). Faúndez (2007c) entrega una lista de los Acantosomátidos de Magallanes. Adicionalmente Carvajal y Faúndez (2013; en prensa) han realizado nuevas contribuciones a la fauna de Acanthosomatidae de la Región.

1 Entomology Department, North Dakota State University, Dept. 7650, P.O. Box 6050; Fargo, ND. USA. mariom. carvajal@gmail.com $\bowtie$

2 Departamento de Zoología Médica, Centro de Estudios en Biodiversidad (CEBCh), Magallanes, 1979, Osorno, Chile 
Planois Signoret, 1864 es un género de Acanthosomatidae que contiene dos especies, Planois gayi (Spinola, 1852) y Planois patagonus Distant, 1911. Este género se distribuye en el sur de Chile y Argentina y ha sido asociado con distintas especies de Nothofagus Blume (Carvajal, 2011). Cabo de Hornos fue aprobado como una reserva de la biosfera por la UNESCO en 2005. El objetivo de esta contribución es describir una nueva especie de Planois de la reserva de la biosfera Cabo de Hornos, en Magallanes, entregar una lista actualizada de los Acantosomátidos de esta región y una clave para su identificación.

\section{MATERIALES Y MÉTODOS}

Las fotografías fueron tomadas con una cámara digital adaptada a un microscopio estereoscópico. En morfología y formato descriptivo seguimos a Faúndez et al. (2014). Las colecciones mencionadas en el texto son las siguientes: Instituto de la Patagonia, Universidad de Magallanes (IPUM), Mariom A. Carvajal (MACC). Todas las medidas se encuentran en milímetros.

\section{RESULTADOS}

Planois smaug sp. nov. (Figs. 1-6, 9, 11)

Descripción general: De forma alargada (Fig. 1), coloración general verde oscuro, con toques rojizos. Punteado de color acaramelado, profundo e irregularmente distribuido en el cuerpo, más denso y grueso en el escutelo.

Cabeza: Forma alargada, paraclípeos redondeados en el extremo anterior, con puntuación fina, dispersa, y estrías transversales; ambas, la puntuación y estrías ausentes en el área adyacente a los ojos. Anteclípeo redondeado, sobrepasando los paraclípeos, inmaculado. Ojos prominentes. Ocelos claros, rojizos (probablemente decolorados por la preservación). Rostrum alcanzando la metacoxa. Sólo se encuentra el primer antenómero en la hembra, que es más largo que la cabeza, claramente sobrepasando ésta, de una tonalidad más oscura que el resto que el cuerpo.

Tórax: Pronoto trapezoide (Fig. 6), con puntuación de igual grosor que en la cabeza en los alrededores, y un poco más gruesa y densa en el centro. Ángulos anterolaterales del pronoto presentan un diente pequeño. Cicatrices inmaculadas, bien definidas, en forma de triángulo recto. Escutelo en forma de triángulo isósceles, con una línea media vertical callosa, más clara que el resto del cuerpo, impuntuada, y con los márgenes laterales rojizos. Puntuación del escutelo más densa a medida que se acerca hacia el ápice. Clavus rojizo con tres corridas paralelas de puntos finos. Corium verde oscuro, y rojizo en la zona adyacente al clavus, con una mancha callosa en el medio, que también presenta coloración rojiza. Puntuación más densa en la mitad interior que en la exterior. Patas con escasa pilosidad en las coxas, trocánter y fémur, y abundante pilosidad de color acaramelado en la tibia y tarsos. Metapleura: Ostíolo alargado; peritrema ostiolar corto, redondeado y carnoso; evaporatoria rugosa, sin puntuación; placa ostiolar lisa.

Abdomen rojizo oscuro, con un par de espiráculos en cada segmento, junto con un par de tricobotrias en una formación callosa justo abajo de cada espiráculo. Conexiva de color amarillento con bandas negras transversales.

Genitalia femenina (Fig. 4): Primer gonocoxito en forma de "D", estriado longitudinalmente, sin puntuación y escasa pilosidad. Segundos gonocoxitos pequeños, casi imperceptibles, redondos. Paraterguitos 9 en forma de triángulo recto; paraterguitos 8 redondeados, anchos lateralmente. Órganos de Pendergrast contiguos, ubicados en los esternitos 6 y 7.

Genitalia masculina (Fig. 2): Pigóforo apicalmente con dos procesos laterales y en el medio con un proceso ampliamente redondeado (Fig. 3). Parámeros (Figs. 5 y 11) aguzados apicalmente, con el ápice redondeado y dirigido hacia el interior; cóncavos en la cara interior en la mitad superior; mayoritariamente convexos en la cara exterior, excepto por una pequeña área cóncava aproximadamente a la mitad de la longitud de la estructura.

Medidas (Hembra en paréntesis): Largo total: 11,38 (14,72); largo de la cabeza: 1,71 $(2,17)$; ancho de la cabeza: $2,52(2,83)$; ancho del pronoto: $5,52(6,15)$; largo del pronoto: 2,39 $(2,95)$; largo del escutelo: 3,08 (3,59); ancho del escutelo: 2, 87 (3, 28); Rostrum: $\mathrm{I}=1.18, \mathrm{II}=1.52$, $\mathrm{III}=1.59, \quad \mathrm{IV}=1.09 ;$ Antenas: $\mathrm{I}=2.59$, resto perdidos. 

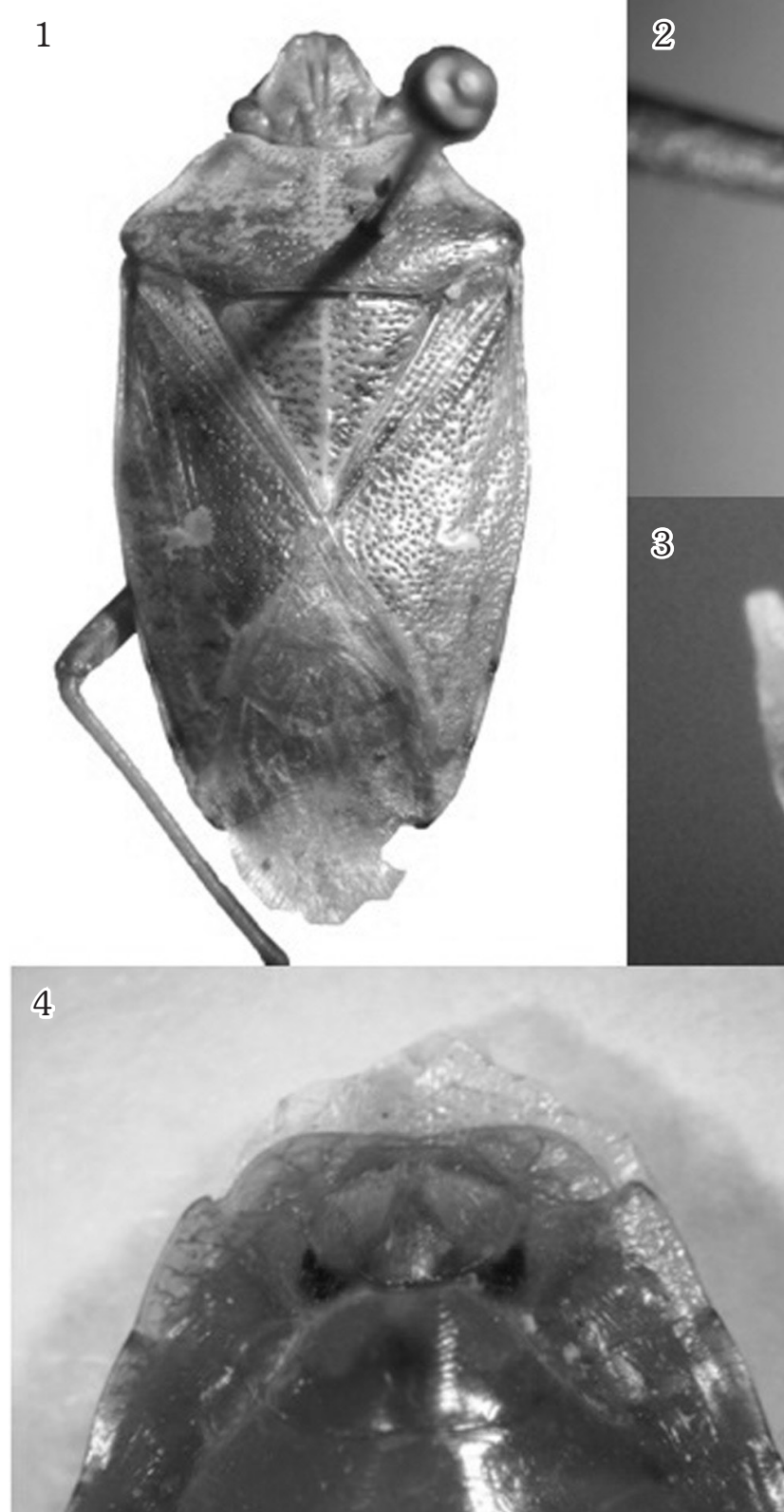

Figs. 1-5. Planois smaug sp. nov. 1, habitus; 2 pigóforo vista caudal, 3 pigóforo vista ventral, 4 genitalia femenina, 5 parámero dentro del pigóforo.
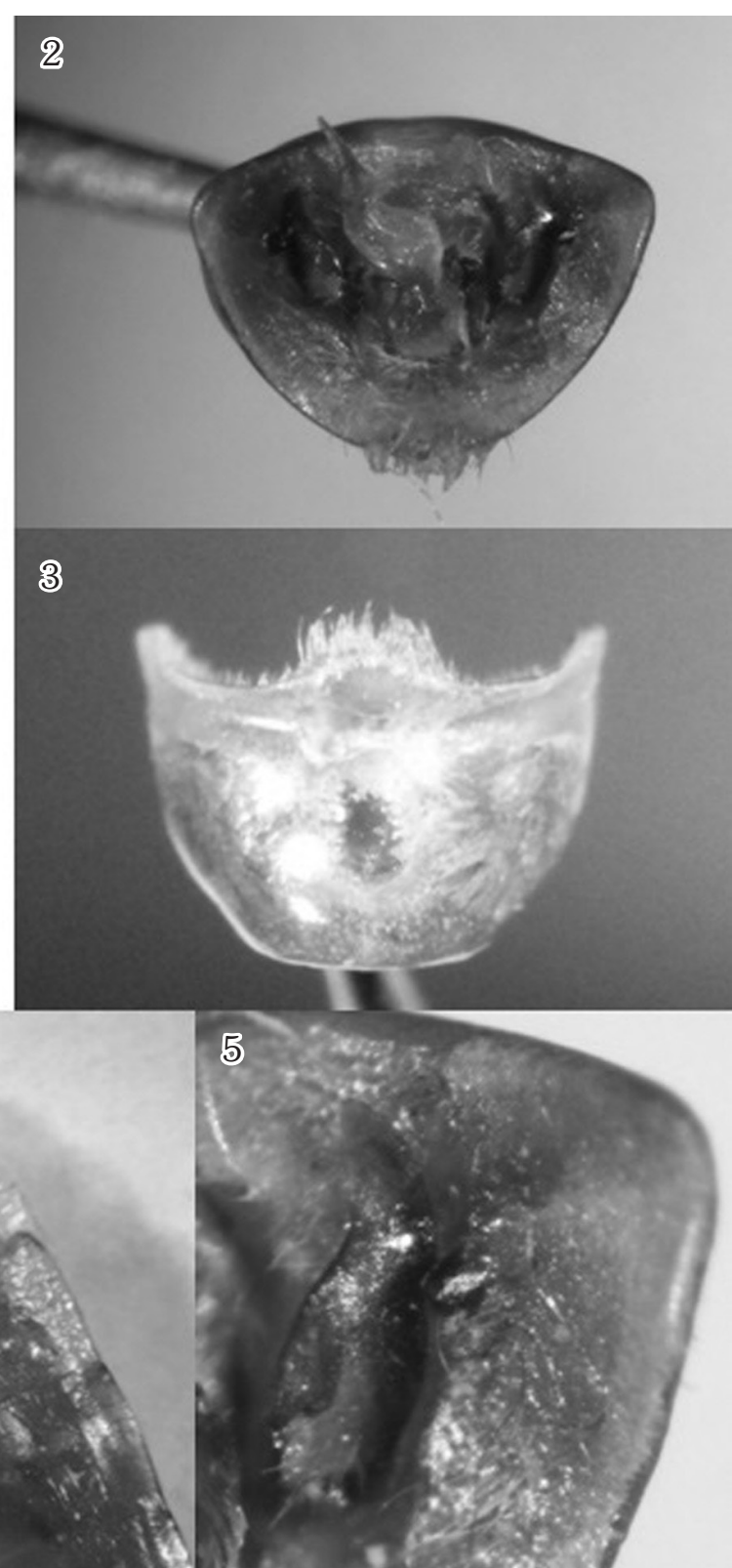

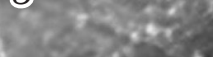

Holotipo: Chile, Isla Navarino, 20-30/ XI/1958, L. E. Peña, leg. 10" (IPUM), pigóforo disectado. Paratipo: mismos datos que el holotipo, 1 을.

Etimología: Smaug, por el personaje ficticio de J. R. R. Tolkien, debido a que esta criatura presentaba un tamaño descomunal, $y$ estuvo dormido en un largo sueño de 60 años; mientras que esta nueva especie sería el chinche más grande en el extremo sur del continente americano, y permaneció sin encontrarse ("dormido") en colecciones por aproximadamente 60 años.

\section{DISCUSIÓN}

Planois smaug se diferencia de Planois 
patagonus por presentar los lados del pronoto cóncavos (Figs. 6 y 8), el ápice del pigóforo más amplio y el anteclípeo más desarrollado; adicionalmente los parámeros de $P$. smaug son más cóncavos en su cara interior y levemente más desarrollados apicalmente (Figs. 11 y 13). De Planois gayi se diferencia por poseer puntuación menos densa, proceso apical del pigóforo más amplio y redondeado (Figs. 9 y 10), parámeros más cóncavos y con zona apical mucho más desarrollada (Figs. 11 y 12) y los lados del pronoto levemente menos inclinados (Figs. 6 y 7). De este modo, el género Planois está representado por 3 especies, $P$. gayi, presente en el sur de Chile desde las regiones del Maule hasta la región de Magallanes (Puerto Edén), Planois patagonus, presente en la zona centro norte de la Patagonia Argentina, y Planois smaug, restringido al extremo austral de la región de Magallanes. Es posible que los registros de $P$. gayi en Puerto Edén correspondan a $P$. samug, lo que debe corroborarse con colectas adicionales. Finalmente para aportar al conocimiento de los Acantosomátidos de la región de Magallanes, entregamos una lista de especies actualizada y una clave de identificación:

Lista de las especies de Acanthosomatidae

Signoret, 1864, de la Región de Magallanes

Familia Acanthosomatidae Signoret, 1864

Subfamilia Ditomotarsinae Signoret, 1864

Tribu Ditomotarsini Kumar, 1974

Género Ditomotarsus Spinola, 1852

1881

Ditomotarsus hyadesi Signoret,

Comentario: Presente en las provincias de Última Esperanza, Magallanes y Cabo de Hornos. Debido a que ataca y daña los frutos de algunas especies utilizadas en la industria alimenticia (calafate, Berberis microphylla, grosella Ribes grossulariae y zarzaparrilla roja Ribes rubrum), debe considerarse su importancia económica (Carvajal \& Faúndez en prensa).
Género Hyperbius Stål, 1868

Hyperbius geniculatus (Signoret,

1864)

Comentario: En la región solamente se ha registrado de la zona norte de la Provincia de Magallanes (Ush Aike) por Faúndez (2007c).

Género Planois Signoret, 1864

Planois gayi (Spinola, 1852)

Comentario: El único registro de esta especie para la Región, corresponde a Puerto Edén (Faúndez, 2007c); sin embargo la presencia de esta especie en Magallanes debe confirmarse con nuevas colectas, ya que este registro podría corresponder a Planois smaug sp. nov.

Planois smaug sp. nov.

Subfamila Bladusinae Kumar, 1974

Tribu Lanopini Kumar, 1974

Género Ea Distant, 1911

Ea australis Distant, 1911

Comentario: Presente en la provincia de Última Esperanza (Faúndez, 2007c; Carvajal et al. 2014).

Género Lanopis Signoret, 1864

Lanopis rugosus Signoret, 1864

Comentario: Presente solo en la isla Wellington, província de Última Esperanza (Faúndez, 2007c).

Género Phorbanta Stål, 1868

Phorbanta variabilis (Signoret,

1864)

Comentario: Presente en las provincias de Magallanes y Última Esperanza (Faúndez, 2007c). Adicionalmente hemos observado 
una serie de ejemplares provenientes del norte de la isla Tierra del Fuego. Hemos observado en agregaciones numerosas de ninfas (hasta 50 ejemplares por planta) atacando frutos de calafate (Berberis microphylla, Berberidaceae), dañando severamente éstos. Dado el uso actual del calafate en la industria alimenticia, la importancia económica de la especie debe considerarse.

\section{Género Sinopla Signoret, 1864}

Sinopla perpunctatus Signoret,

\section{4}

Comentario: Presente en las provincias de Última Esperanza, Magallanes y Cabo de Hornos, es junto con Ditomotarsus hyadesi uno de los Acantosomátidos más comunes de la Región.

1864

\section{Sinopla humeralis Signoret,}

Comentario: Presente sólo en la comuna de Última Esperanza, específicamente en el Parque Nacional Torres del Paine (Carvajal \& Faúndez,

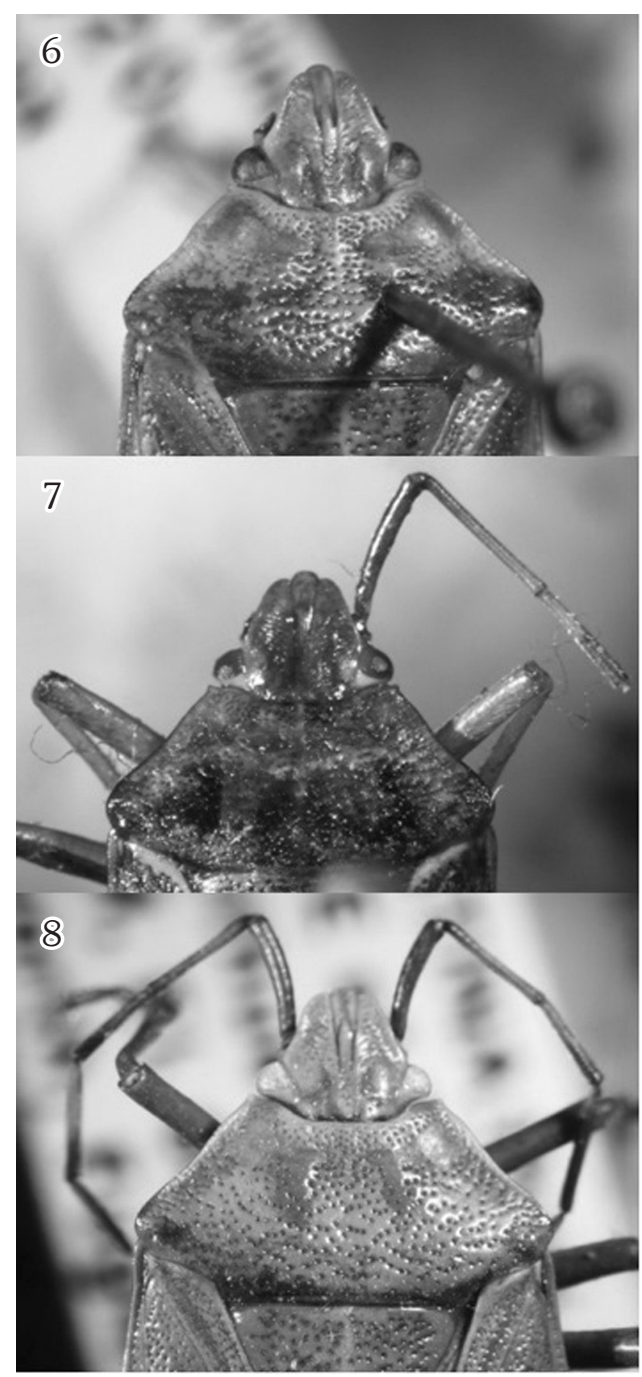

9

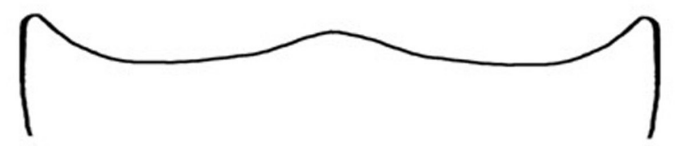

10

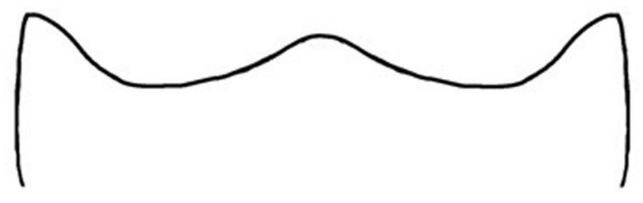

11

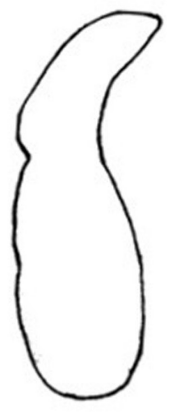

12

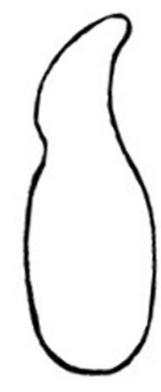

13

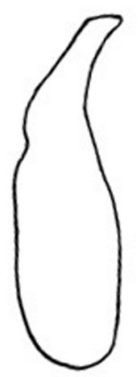

Fig. 6. Planois smaug sp. nov, detalle de cabeza y pronoto; Fig. 7. Planois gayi (Spinola, 1852), detalle de cabeza y pronoto; Fig. 8. Planois patagonus Distant, 1911, detalle de cabeza y pronoto; Fig. 9. P. smaug, borde posterior del pigóforo en vista ventral; Fig. 10. P. gayi, borde posterior del pigóforo en vista ventral; Fig. 11. P. smaug, parámero en posición natural; Fig. 12. P. gayi, parámero en posición natural; Fig. 13. P. patagonus, parámero en posición natural. 
2013).

Clave de identificación para las especies de Acanthosomatidae de la Región de Magallanes

1(10) Con tubérculo o espina media abdominal.

2(3) Paraclípeos afilados apicalmente.

3(2) Paraclípeos no afilados apicalmente.

Phorbanta variabilis (Signoret, 1864)

4(5) Lados del pronoto cóncavos....

Lanopis rugosus Signoret, 1864

5(4) Lados del pronoto rectos o convexos. Ea australis Distant, 1911

6(7) Pronoto trapezoide (Género Sinopla) 8

7(6) Pronoto subtriangular o deltoide.

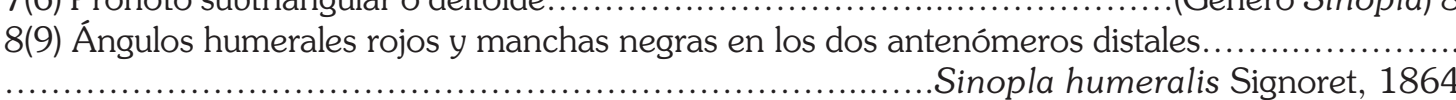

9(8) Pronoto y antenas unicolores. Sinopla perpunctatus Signoret, 1864

10(1) Sin tubérculo o espina media abdominal.... ...11

11(14) Primer antenómero tanto o más largo que la cabeza. (Género Planois) 12

12(13) Puntuación más densa, parámeros con el lóbulo apical poco desarrollado, proceso apical del pigóforo más afilado (Fig. 10) Planois gayi (Spinola, 1852)

13(12) Puntuación menos densa, parámeros con el lóbulo apical más desarrollado y curvado hacia el interior, proceso apical del pigóforo más amplio y redondeado (Fig. 9).

Planois smaug sp. nov.

14(11) Primer antenómero más corto que la cabeza.

15(16) Márgenes laterales de la cabeza pronunciadamente cóncavos, coloración general negra con diseños marfileños. Hyperbius geniculatus (Signoret, 1864)

16(15) Márgenes de la cabeza rectos o convexos y coloración verde oscuro o parda. Ditomotarsus hyadesi Signoret, 1881

\section{LITERATURA CITADA}

Carvajal, M. A (2011). Nuevos registros para Planois gayi (Spinola, 1852) (Hemiptera: Acanthosomatidae) en la Región de la Araucanía, Chile. Boletín de la Sociedad Entomológica Aragonesa, 49, 357-358.

Carvajal, M. A. \& Faúndez, E. I. (2013). Rediscovery of Sinopla humeralis Signoret, 1864 (Hemiptera: Heteroptera: Acanthosomatidae). Zootaxa, 367(2), 190196.

Carvajal, M. A. \& Faúndez, E. I. (en prensa). Revalidation of Ditomotarsus hyadesi Signoret, 1881 (Hemiptera: Heteroptera: Acanthosomatidae), with notes on its natural history. Biodiversity and Natural History.

Carvajal, M. A., Faúndez, E. I. \& Rider, D. A. (2014). New data on the genus Ea Distant, 1911 (Hemiptera: Heteroptera: Acanthosomatidae), with description of a new species from Chile. Anales del Instituto de la Patagonia, 42(2), 53-58.

Faúndez, E. I. (2007a). Notes on the biology of Ditomotarsus punctiventris Spinola, 1852 (Hemiptera: Acanthosomatidae) in the Magellan region, and comments about the crypsis in Acanthosomatidae. Anales del Instituto de la Patagonia, 35(2), 67-70.

Faúndez, E. I. (2007b). Asociación críptica entre Sinopla perpunctatus Signoret, 1863 (Acanthosomatidae: Hemiptera) y el Nirre Nothofagus antarctica (G. Forster) Oersted (Fagaceae) en la Región de Magallanes (Chile). Boletín de la Sociedad Entomológica Aragonesa, 40, 563-564.

Faúndez, E. I. (2007c). Lista de las especies de Acanthosomatidae (Insecta: Hemiptera) de la región de Magallanes (Chile). Anales del Instituto de la Patagonia, 35(1), 75-78.

Faúndez, E. I., Osorio, G. A., Bahamondez, C. P. \& Monsalve, E. A. (2009). Comportamiento gregario en los hábitos sexuales de 
Ditomotarsus punctiventris Spinola, 1852 (Hemiptera:Heteroptera:Acanthosomatidae). Boletín de Biodiversidad de Chile, 1(1), 1518.

Faúndez, E. I. \& Osorio, G. A. (2010). New data on the biology of Sinopla perpunctatus Signoret, 1864 (Hemiptera: Heteroptera: Acanthosomatidae). Boletín de Biodiversidad de Chile, 3, 24-31.
Faúndez, E. I., Carvajal, M. A., \& Rider, D. A. (2014). Archaeoditomotarsus crassitylus, gen. and sp. nov. from Chile. Zootaxa, 3860(1),87-91.

Tsai, J. \& Rédei, D. (2015). The identity of Acanthosoma vicinum, with proposal of new genus and species level synonymy (Hemiptera: Heteroptera: Acanthosomatidae). Zootaxa, 3936(3), 375-386. 
